\title{
Effect of target surface on elastic properties of fast fullerenes
}

\author{
S. Wethekam, ${ }^{1}$ M. Busch, ${ }^{1, *}$ Ch. Linsmeier, ${ }^{2}$ and H. Winter ${ }^{1}$ \\ ${ }^{1}$ Institut für Physik, Humboldt-Universität zu Berlin, Newtonstrasse 15, D-12489 Berlin, Germany. \\ ${ }^{2}$ Max-Planck-Institut für Plasmaphysik, EURATOM Association, Boltzmannstr. 2, D-85748 Garching, Germany.
}

$\mathrm{C}_{60}^{+}$fullerenes with $\mathrm{keV}$ energies are scattered at grazing angles of incidence from atomically clean and flat $\mathrm{LiF}(001), \mathrm{KCl}(001), \mathrm{Al}(001), \mathrm{Be}(0001), \mathrm{Ni}(110)$ surfaces as well as $\mathrm{p}(2 \times 1)$ and $\mathrm{p}(3 \times 1)$ oxygen superstructures on $\mathrm{Ni}(110)$. The elastic properties of $\mathrm{C}_{60}$ are derived from a comparison of experimental data with 3D molecular dynamics simulations for different interaction potentials. In terms of a simple model for the hybridization of $\mathrm{C}_{60}$ with the surface, we find evidence for a close relation between electronic structure of the surface and elasticity of $\mathrm{C}_{60}$.

PACS numbers: 79.20.Rf, 34.35.+a, 79.60.Bm, 79.20.-m, 78.30.Na, 61.85.+p

The elastic properties of carbon nanostructures play an important role for applications in composite materials, nanomechanical devices, or molecular electronics [1-7]. In such configurations, carbon nanostructures will be embedded, supported, or in contact with a matrix of material, that will affect the properties of the carbon nanostructure. Prominent examples are strong covalent interactions of fullerenes with surfaces such as $\mathrm{Si}(100)$, $\mathrm{Ni}(110)$, or $\mathrm{Pt}(111)$ resulting in dissociation of fullerenes in thermal desorption spectroscopy (TDS) experiments or charge transfer processes inducing semiconductorconductor phase transitions [8-10]. By an appropriate choice of the surrounding material, these interactions might be exploited for an optimized performance of the building blocks of future carbon nanostructure devices or materials.

From a fundamental point of view, it is important to provide experimental data on the properties of single carbon nanostructures in different environments. Here, we focus on the elastic properties of the prototype of a carbon nanostructure, $\mathrm{C}_{60}$ [11], in front of single crystal surfaces for deformation energies up to some $10 \mathrm{eV}$. As the ultimate experimental tool, a single molecule vise with two opposing non-deformable surfaces, cannot be realized, we performed scattering experiments in the surface channeling regime, i.e. under grazing incidence, where the deformation of the surface is negligible due to the small energy transfer to the surface in small-angle scattering events $[12,13]$. The velocity of the molecules is still sufficiently slow in order to guarantee an adiabatic regime for the interaction with the surface [14]. Via the incident energy and the angle of incidence, the molecule can be exposed to well-defined deformation energies. The elastic properties of the molecules are studied by means of the kinetic energy of outgoing fullerenes. In the case of a stiff/soft molecule, a small/large amount of the energy is transferred to internal degrees of freedom and the outgoing kinetic energy is large/small.

During the last two decades, a fair number of studies have been devoted to elastic properties, stability, fragmentation, and charge transfer during scattering of hyperthermal fullerenes from surfaces [15-22]. However, for angles of incidence $\Phi_{\text {in }} \geq 10^{\circ}$ with respect to the surface, the deformation of the surface was considerable and affected the kinetic energies of outgoing molecules. First experiments under grazing angles of incidence of $\Phi_{\text {in }} \approx 1^{\circ}$ with a negligible energy transfer to the surface were recently reported by Kimura et al. [23-25]. For the full range of energies for the motion normal to a $\mathrm{KCl}(001)$ surface of up to $E_{\perp}^{\text {in }}=E \sin ^{2} \Phi_{\text {in }}=20 \mathrm{eV}$ ( $E$ : total energy), the $\mathrm{C}_{60}$ molecules where scattered fully elastically.

Motivated by this work, we have studied grazing scattering of $\mathrm{C}_{60}$ molecules from an $\mathrm{Al}(001)$ surface $[26,27]$ and found pronounced differences to the data of Kimura et al. for the insulator $\mathrm{KCl}(001)$. For normal energies $E_{\perp} \geq 7 \mathrm{eV}$, considerable energy losses $\Delta E_{\perp}^{\text {loss }}$ for the motion normal to the surface were observed. An analysis of fragment spectra revealed that $\Delta E_{\perp}^{\text {loss }}$ was completely transferred to internal excitations of the fullerenes. 3D molecular dynamics simulations showed that $\Delta E_{\perp}^{\text {loss }}$ does not depend on the interaction potential of the molecule with the surface, but is related to internal elastic properties of the molecule $[26,27]$. Therefore, the differences in $\Delta E_{\perp}^{\text {loss }}$ for scattering of $\mathrm{C}_{60}$ from $\mathrm{KCl}(001)$ and $\mathrm{Al}(001)$ might to stem from different elastic properties of the fullerene in front of both surfaces.

In order to clear up this important feature, we have extended our study on the elastic properties of $\mathrm{C}_{60}$ during grazing scattering from $\mathrm{LiF}(001), \mathrm{KCl}(001), \mathrm{Al}(001)$, $\mathrm{Be}(0001), \mathrm{Ni}(110)$ surfaces as well as $\mathrm{p}(2 \times 1)$ and $\mathrm{p}(3 \times 1)$ oxygen superstructures on $\mathrm{Ni}(110)$, so that the electronic properties of the target surfaces are varied over a wide range. The data are compared to 3D molecular dynamics simulations for different interaction potentials for the $\mathrm{C}$ atoms of the $\mathrm{C}_{60}$ molecules and for the interaction of the fullerenes with the surface. We find that electronic structure of the surface and elasticity of the fullerene are closely related. A simple model of hybridization of $\mathrm{C}_{60}$ with the surface, provides a qualitative understanding of the different elastic properties of the fullerene in front of different surfaces.

In our experiments, we have scattered $\mathrm{C}_{60}^{+}$molecular ions with energies $E$ up to some $10 \mathrm{keV}$ under grazing angles of incidence $\Phi_{\text {in }}$ of typically $1^{\circ}$ from atomically clean 
and flat surfaces. Scattering proceeds in the surface channeling regime $[12,13]$, where the motions parallel and normal to the surface are widely decoupled. Whereas the parallel motion takes place with $\mathrm{keV}$ energies $E_{\|}=$ $E \cos ^{2} \Phi_{\text {in }} \approx E$, the interaction with the surface is governed by the slow normal motion with about three orders of magnitude smaller energy $E_{\perp}^{\text {in }}=E \sin ^{2} \Phi_{\text {in }} \sim 10^{-3} E$, i.e. in the regime of $\mathrm{eV}$.

The molecules were produced by evaporation of fullerene powder (SES research, Houston, TX) in an electron cyclotron resonance (ECR) ion source (NanoganPantechnique, Caen, France) in Ar atmosphere. After extraction at $2.8 \mathrm{keV}$, the molecular ions were massseparated in a $90^{\circ}$ deflection magnet and accelerated to their final energy $E$. Before impacting the surface, the divergence of the beam was reduced to about $0.03^{\circ}$ by means of a slit system. The surfaces were mounted on a precision manipulator in an ultra-high vacuum (UHV) chamber with a base pressure of some $10^{-11}$ mbar and were prepared by cycles of grazing sputtering with $25 \mathrm{keV}$ $\mathrm{Ar}^{+}$ions and subsequent annealing. The quality of the surfaces was checked by ion beam and standard surface analytical tools [13]. The oxygen superstructures on $\mathrm{Ni}(110)$ were prepared by the controlled adsorption of $\mathrm{O}_{2}$ at elevated temperatures (for details see Ref. [28]). The scattered projectiles of different charge were dispersed in an electric field and detected $66 \mathrm{~cm}$ behind the target with a position sensitive microchannel plate detector (DLD40, Roentdek, Kelkheim-Ruppertshain, Germany). For details on the experimental setup see Ref. [26].

In Fig. 1, we show polar angular distributions for scattering of $E=10 \mathrm{keV} \mathrm{Ar}^{0}$ atoms (solid curves) and $\mathrm{C}_{60}^{+}$ ions (dashed curves: outgoing $\mathrm{C}_{60}^{0}$, dotted curves: outgoing $\mathrm{C}_{60}^{+}$) for different incident normal energies $E_{\perp}^{\text {in }}$ from clean $\mathrm{Ni}(110)$ (left panel) and from a $\mathrm{p}(3 \times 1)$ oxygen superstructure on $\mathrm{Ni}(110)$ (right panel). The corresponding outgoing normal energies $E_{\perp}^{\text {out }}=E \sin ^{2} \Phi_{\text {out }}$ are given in the upper scale. The angular distributions for $\operatorname{Ar}^{0}$ atoms serve as a reference for elastic scattering, where the width is determined by thermal displacements of target atoms [13]. The distributions for outgoing neutral and charged fullerenes coincide. The fragmentation of the fullerenes can be checked for charged molecules via deflection in an electric field. We do not find any indication for a fragmentation at the surface in the investigated range of $E_{\perp}$. However, postcollisional and delayed fragmentations might be possible and slightly broaden the distributions, but do not shift the position of the maximum due to their isotropy [15, 26, 34].

At small normal energies $E_{\perp}^{\text {in }} \leq 2 \mathrm{eV}, \mathrm{C}_{60}^{+}$molecular ions are scattered elastically from $\mathrm{Ni}(110)$, whereas for $E_{\perp}^{\text {in }} \geq 3 \mathrm{eV}$, the fullerenes are scattered subspecularly with a mean outgoing normal energy of $E_{\perp}^{\text {out }} \approx 3 \mathrm{eV}$. For the largest normal energy of $E_{\perp}^{\text {in }} \approx 12 \mathrm{eV}$, this corresponds to a normal energy loss $\Delta E_{\perp}^{\text {loss }}=E_{\perp}^{\text {in }}-E_{\perp}^{\text {out }} \approx 9 \mathrm{eV}$. For the oxygen superstructure $\mathrm{p}(3 \times 1) \mathrm{O} / \mathrm{Ni}(110$, i.e. for less

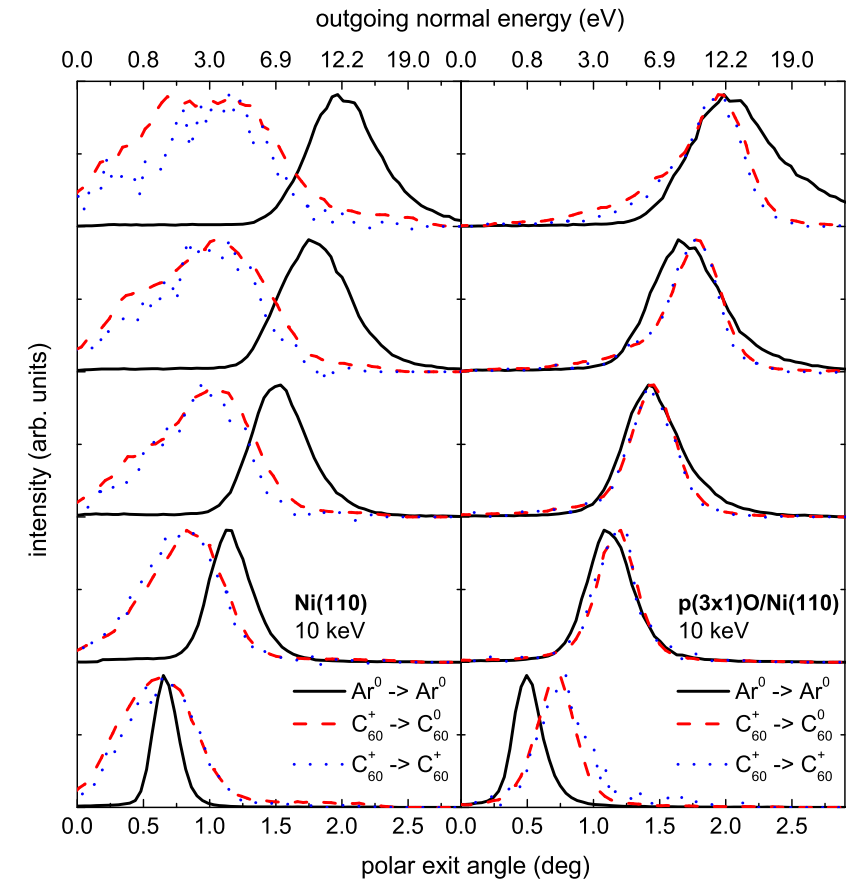

FIG. 1. (Color online) Normalized polar angular distributions for scattering of $E=10 \mathrm{keV} \mathrm{Ar}^{0}$ atoms (solid curves) and $\mathrm{C}_{60}^{+}$molecular ions (dashed curves: outgoing $\mathrm{C}_{60}^{0}$, dotted curves: outgoing $\left.\mathrm{C}_{60}^{+}\right)$with different angles of incidence $\Phi_{\text {in }}$ from $\mathrm{Ni}(110)$ (left panel) and $\mathrm{p}(3 \times 1) \mathrm{O} / \mathrm{Ni}(110)$ (right panel). Upper scale: polar exit angle $\Phi_{\text {out }}$ (lower scale) converted to outgoing normal energy $E_{\perp}^{\text {out }}=E \sin ^{2} \Phi_{\text {out }}$.

than one monolayer oxygen on the $\mathrm{Ni}(110)$ surface, the behavior is completely different. The molecules are scattered elastically for normal energies up to $E_{\perp}^{\text {in }}=13 \mathrm{eV}$. In passing we note that the slight shift of the distributions for the fullerenes and the $\mathrm{Ar}^{0}$ atoms for the smallest normal energy is a result of the attractive force owing to image charge interactions $[29,30]$ on the incident path prior to neutralization. As the outgoing charged fullerenes are formed via a delayed ionization after the collision, the distributions for neutral and charged outgoing fullerenes are not shifted with respect to each other [15, 26].

For a systematic analysis, we show in Fig. 2 the elasticity ratio $E_{\perp}^{\text {out }} / E_{\perp}^{\text {in }}$, i.e. the ratio of the outgoing $E_{\perp}^{\text {out }}$ and the incident normal energy $E_{\perp}^{\text {in }}$, derived from the maxima of polar angular distributions as function of $E_{\perp}^{\text {in }}$ for scattering of $\mathrm{C}_{60}^{+}$molecular ions from $\mathrm{LiF}(001), \overrightarrow{\mathrm{KCl}}(001), \mathrm{Al}(001), \mathrm{Be}(0001), \mathrm{Ni}(110)$, $\mathrm{p}(2 \times 1) \mathrm{O} / \mathrm{Ni}(110)$, and $\mathrm{p}(3 \times 1) \mathrm{O} / \mathrm{Ni}(110)$ surfaces. The data reveals pronounced differences for $E_{\perp}^{\text {out }} / E_{\perp}^{\text {in }}$ and the normal energy loss $\Delta E_{\perp}^{\text {loss }}$ for different surfaces. Note that less than a monolayer of oxygen on a $\mathrm{Ni}(110)$ surface is sufficient to establish a similar behavior as observed for insulator surfaces.

In order to explore the physical mechanism, we performed 3D molecular dynamics simulations for different potentials for the $\mathrm{C}-\mathrm{C}$ and the fullerene-surface interac- 


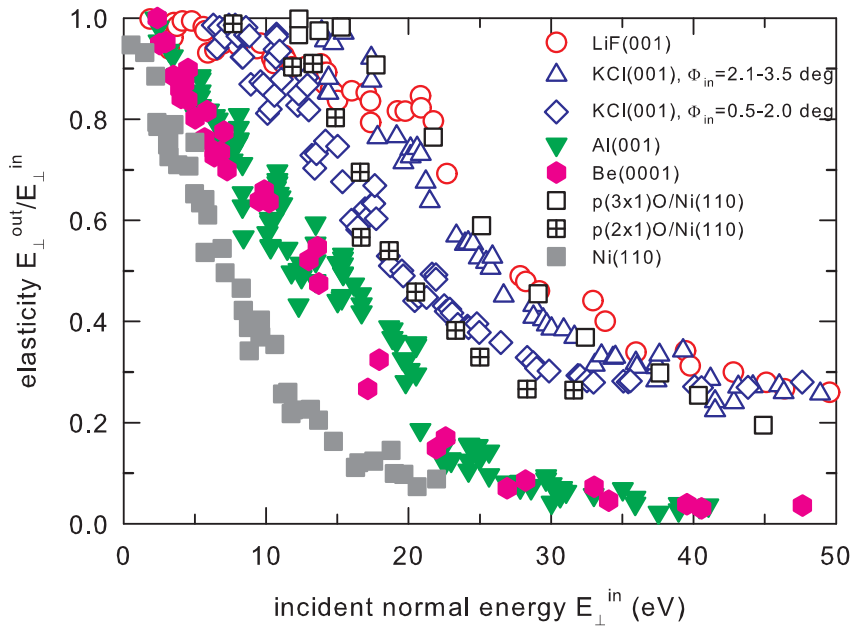

FIG. 2. (Color online) Elasticity $E_{\perp}^{\text {out }} / E_{\perp}^{\text {in }}$ as function of incident normal energy $E_{\perp}^{\text {in }}$ for scattering of $\mathrm{C}_{60}^{+}$molecular ions from $\mathrm{LiF}(001), \mathrm{KCl}(001), \mathrm{Al}(001), \mathrm{Be}(0001), \mathrm{Ni}(110), \mathrm{p}(2 \times 1)$ and $\mathrm{p}(3 \times 1) \mathrm{O} / \mathrm{Ni}(110)$ surfaces as indicated.

tion. Thermal displacements of target atoms were included in the framework of the Debye model and internal excitations in the ion source of incident fullerenes by about $35 \mathrm{eV}$ was incorporated. For details on the simulation concepts and numerical routines, we refer to Refs. $[26,27]$. For each set of parameters, 30 trajectories were calculated and the mean values are shown by the symbols in Fig. 3 together with the experimental data from Fig. 2 for the two extreme cases, $\operatorname{LiF}(001)$ (full circles) and $\mathrm{Ni}(110)$ (full squares). We apply the Tersoff [31] (small symbols in Fig. 3) as well as the more recent Albe potential [32] (large symbols in Fig. 3) for the interaction of the $\mathrm{C}$ atoms of the fullerene. The results for both potentials coincide. In case of the insulator surfaces $\mathrm{LiF}(001)$ and $\mathrm{KCl}(001)$, the molecules are not or only partly neutralized at the surface $[23-25,33,34]$, so that we performed simulations for neutral $\mathrm{C}_{60}^{0}$ and charged $\mathrm{C}_{60}^{+}$fullerenes including intra-molecule Coulomb interactions and the classical image charge interaction with the surface.

The interaction of the molecule with the surface is implemented by a Molière potential with modified Firsov screening length proposed by O'Connor and Biersack [35]. In addition, for the $\mathrm{Al}(001)$ surface, we show results using potentials from literature ("attr. S. W.": [36], "attr. A. P.-J.": attractive part of potential from [37] for $\mathrm{Au}(111)$ taking into account the relative shifts of jellium edges of $\mathrm{Al}(001)$ and $\mathrm{Au}(111))$. In order to exclude a possible effect of the modified bond-order of the $\mathrm{C}$ atoms of the fullerene due to the interaction with the surface, we also show a calculation for $\mathrm{Al}(001)$ ("attr. incl. bondorder"), where the $\mathrm{Al}$ atoms were treated as $\mathrm{C}$ atoms in terms of the Tersoff potential. In order to avoid a complete desintegration of the molecules at the surface, the attractive part of the fullerene surface interaction had to

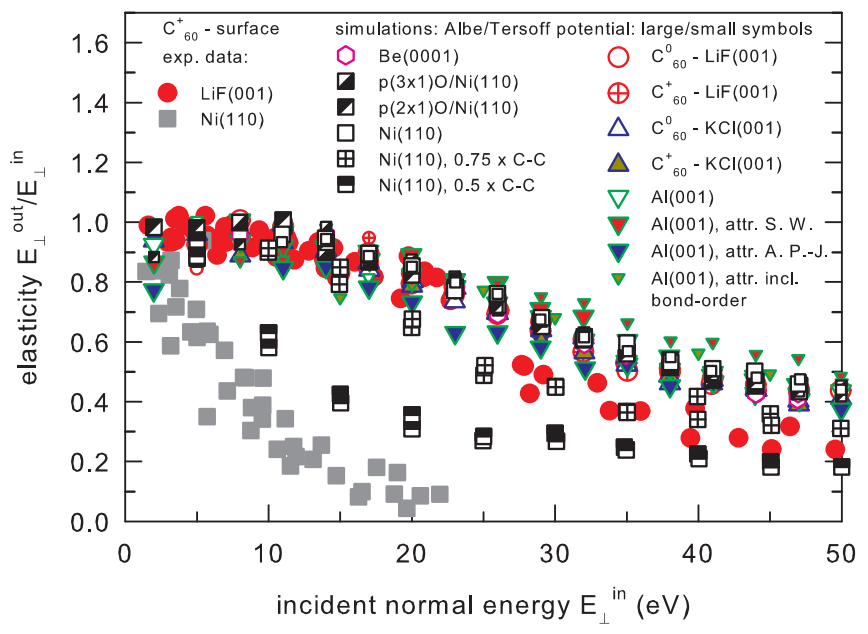

FIG. 3. (Color online) Simulated elasticity $E_{\perp}^{\text {out }} / E_{\perp}^{\text {in }}$ as function of incident normal energy $E_{\perp}^{\text {in }}$ for scattering of $\mathrm{C}_{60}^{0}$ and $\mathrm{C}_{60}^{+}$from indicated surfaces for Albe and Tersoff potential for different parameter sets. Full circles/squares: experimental data for $\mathrm{LiF}(001) / \mathrm{Ni}(110)$ (cf. Fig. 2). For details see text.

be reduced by a factor of two. The bond-order was kept constant.

In good accord with our analysis for an $\mathrm{Al}(001)$ and a $\mathrm{KCl}(001)$ surface $[26,27,34]$, different surface structures, interaction potentials of the fullerene with the surfaces, or charge of the fullerene do not affect the outcome of the simulations. The results fall on one curve and reproduce the experimental data for the surfaces with the smallest normal energy losses $\Delta E_{\perp}^{\text {loss }}$, i.e. the largest elasticity $E_{\perp}^{\text {out }} / E_{\perp}^{\text {in }}, \mathrm{LiF}(001)$ and $\mathrm{p}(3 \times 1) \mathrm{O} / \mathrm{Ni}(110)$, on a near-quantitative level. Therefore, we conclude that the differences in the elasticity for the different surfaces are not related to differences in the interaction potentials of the fullerene with the surface nor to the charge of the fullerene in front of the surface.

The only reasonable possibility to explain the pronounced normal energy losses during scattering from a metal surface seems to be an influence of the surface on the internal elastic properties of the molecule. In Fig. 3 , we have included calculations for intra-molecular $\mathrm{C}-\mathrm{C}$ interactions reduced by a factor of 0.75 (labeled as " 0.75 $\times$ C-C") and 0.5 (labeled as " $0.5 \times$ C-C"), which result in strongly reduced elasticity.

In Fig. 4, we show an energy diagram for a $\mathrm{C}_{60}$ molecule (HOMO: highest occupied molecular orbital, LUMO: lowest unoccupied molecular orbital) [1] and different surfaces [38, 39] arranged as function of decreasing elasticity, i.e. increasing normal energy loss $\Delta E_{\perp}^{\text {loss }}$, from left to right. We find a clear-cut correlation of normal energy loss and electronic band structure of the surface. The smaller the energy defect between the electronic states of fullerene and surface, the stronger is the reduction of the elasticity of the fullerene. Of particular interest is the case of $\mathrm{KCl}(001)$, where we have observed 


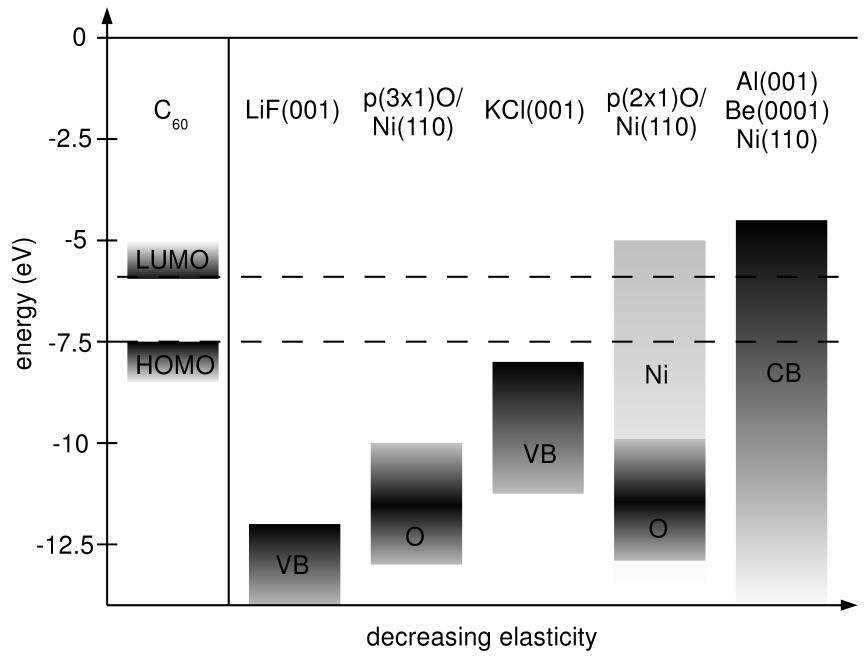

FIG. 4. Energy diagram for a $\mathrm{C}_{60}$ molecule and different indicated surfaces arranged as function of decreasing elasticity, i.e. increasing normal energy loss $\Delta E_{\perp}^{\text {loss }}$. $\mathrm{VB}(\mathrm{CB})$ : valence (conduction) band. O: oxygen adsorbate states. Ni: $\mathrm{CB}$ of $\mathrm{Ni}(110)$ for more open $\mathrm{p}(2 \times 1)$ oxygen adsorbate structure.

slightly larger normal energy losses for smaller angles of incidence $\Phi_{\text {in }}$ at same normal energy $E_{\perp}^{\text {in }}$ in Fig. 2. As the same normal energy for smaller angles of incidence is reached for larger beam energies $E$, this might be a consequence of a kinematically induced broadening [13, 34, 40] of the upper edge of the valence band ( $\mathrm{VB}$ ) of $\mathrm{KCl}(001)$, which reduces the energy defect with the HOMO. As the $\mathrm{p}(2 \times 1) \mathrm{O} / \mathrm{Ni}(110)$ superstructure has a smaller oxygen density than the $\mathrm{p}(3 \times 1) \mathrm{O} / \mathrm{Ni}(110)$ superstructure, the interaction of the fullerene with electronic states of the substrate is stronger than for $\mathrm{p}(3 \times 1) \mathrm{O} / \mathrm{Ni}(110)$ indicated by the light gray area for $\mathrm{p}(2 \times 1) \mathrm{O} / \mathrm{Ni}(110)$ in Fig. 4 .

In summary, we attribute the modification of internal elastic properties of the fullerenes to a hybridization of molecular electronic states with electronic states of the surface. This hybridization results in reduced elasticities, most prominent for metal surfaces. In spite of different approaches, our experiments are in good accord with the classification of fullerene-surface interaction strengths by Maxwell et al. [8] based on TDS studies of thin fullerene films. We hope to trigger further studies on this fundamental aspect of molecule-surface interactions. Compared to TDS studies, our data provides complementary detailed information, as we provide complete elasticity curves, where the distance of closest approach and the deformation of the fullerene at the surface are varied over a large parameter range.

Financial support by the Deutsche Forschungsgemeinschaft (Project No. Wi 1336) and fruitful discussions with K. Kimura (Kyoto), E. Kolodney (Haifa), H. Zettergren and H. Cederquist (Stockholm) are gratefully acknowledged. We thank K. Maass, A. Schüller, U. Specht, and G. Lindenberg for their assistance in the preparation of the experiments.

* Corresponding author: mbusch@physik.hu-berlin.de

[1] M. S. Dresselhaus, G. Dresselhaus, and P. C. Eklund, Science of Fullerenes and Carbon Nanotubes (Academic Press, San Diego, 1996).

[2] R. H. Baughman, A. A. Zakhidov, and W. A. de Heer, Science 297, 787 (2002).

[3] F. Rosei, M. Schunack, Y. Naitoh, P. Jiang, A. Gourdon, E. Laegsgaard, I. Stensgaard, C. Joachim, and F. Besenbacher, Prog. Surf. Sci. 71, 95 (2003).

[4] J. A. Åström, A. V. Krasheninnikov, and K. Nordlund, Phys. Rev. Lett. 93, 215503 (2004).

[5] M. P. Anantram and F. Leonard, Rep. Prog. Phys. 69, 507 (2006).

[6] H. Dai, J. H. Hafner, A. G. Rinzler, D. T. Colbert, and R. E. Smalley, Nature 384, 147 (1996).

[7] J. H. Hafner, C. L. Cheung, A. T. Woolley, and C. M. Lieber, Prog. Biophys. and Mol. Biol. 77, 73 (2001).

[8] A. J. Maxwell, P. A. Brühwiler, D. Arvanitis, J. Hasselström, M. K.-J. Johansson, and N. Mårtensson, Phys. Rev. B 57, 7312 (1998).

[9] S. Modesti, S. Cerasari, and P. Rudolf, Phys. Rev. Lett. 71, 2469 (1993).

[10] A.-C. Wade, S. Lizzit, L. Petaccia, A. Goldoni, D. Diop, H. Üstünel, S. Fabris, and S. Baroni, J. Chem. Phys. 132, 234710 (2010).

[11] H. W. Kroto, J. R. Heath, S. C. OBrien, R. F. Curl, and R. E. Smalley, Nature 318, 162 (1985).

[12] D. S. Gemmell, Rev. Mod. Phys. 46, 129 (1974).

[13] H. Winter, Phys. Rep. 367, 387 (2002).

[14] P. M. Echenique, R. Berndt, E. V. Chulkov, T. Fauster, A. Goldmann, and U. Höfer, Surf. Sci. Rep. 52, 219 (2004).

[15] E. E. B. Campbell, Fullerene Collision Reactions (Kluwer Academic, London, 2003).

[16] T. Lill, H. G. Busmann, F. Lacher, and I. V. Hertel, Chem. Phys. 193, 199 (1995).

[17] R. D. Beck, J. Rockenberger, P. Weis, and M. M. Kappes, J. Chem. Phys. 104, 3638 (1996).

[18] M. Hillenkamp, J. Pfister, M. M. Kappes, and R. P. Webb, J. Chem. Phys. 111, 10303 (1999).

[19] M. Hillenkamp, J. Pfister, and M. M. Kappes, J. Chem. Phys. 114, 10457 (2001).

[20] E. Kolodney, B. Tsipinyuk, A. Bekkerman, and A. Budrevich, Nucl. Instrum. Meth. Phys. Res. B 125, 170 (1997).

[21] A. Bekkerman, B. Tsipinyuk, and E. Kolodney, Phys. Rev. B 61, 10463 (2000).

[22] A. Kaplan, A. Bekkerman, B. Tsipinyuk, and E. Kolodney, Phys. Rev. B 79, 233405 (2009).

[23] S. Tamehiro, T. Matsushita, K. Nakajima, M. Suzuki, and K. Kimura, Nucl. Instr. Meth. Phys. Res. B 256, 16 (2007).

[24] T. Matsushita, K. Nakajima, M. Suzuki, and K. Kimura, Phys. Rev. A 76, 032903 (2007).

[25] K. Kimura, T. Matsushita, K. Nakajima, and M. Suzuki, Nucl. Instr. Meth. Phys. Res. B 267, 2638 (2009).

[26] S. Wethekam and H. Winter, Phys. Rev. A 76, 032901 (2007). 
[27] S. Wethekam and H. Winter, Vacuum 82, 895 (2008).

[28] S. Wethekam, M. Busch, and H. Winter, Surf. Sci. 603, 209 (2009).

[29] S. Wethekam, H. Winter, H. Cederquist, and H. Zettergren, Phys. Rev. Lett. 99, 037601 (2007).

[30] S. Wethekam, H. Zettergren, C. Linsmeier, H. Cederquist, and H. Winter, Phys. Rev. B 81, 121416 (2010).

[31] J. Tersoff, Phys. Rev. Lett. 61, 2879 (1988).

[32] P. Erhart and K. Albe, Phys. Rev. B 71, 035211 (2005).

[33] S. Wethekam and H. Winter, Nucl. Instr. Meth. Phys. Res. B 269, 1179 (2011).

[34] S. Wethekam, J. Merck, M. Busch, and H. Winter, Phys. Rev. B 83, 085423 (2011).
[35] D. J. O'Connor and J. P. Biersack, Nucl. Instr. Meth. Phys. Res. B 15, 14 (1986).

[36] S. Wethekam, A. Schüller, and H. Winter, Nucl. Instr. Meth. Phys. Res. B 258, 68 (2007).

[37] Á. J. Pérez-Jiménez, J. J. Palacios, E. Louis, E. SanFabián, and J. A. Vergés, Chem. Phys. Chem. 4, 388 (2003).

[38] A. Goldmann and E. E. Koch, eds., Landolt-Börnstein, Vol. III 23a (Springer-Verlag, Berlin, 1989).

[39] F. Besenbacher and J. K. Norskov, Prog. Surf. Sci. 44, 5 (1993).

[40] J. Los and J. J. C. Geerlings, Phys. Rep. 190, 133 (1990). 